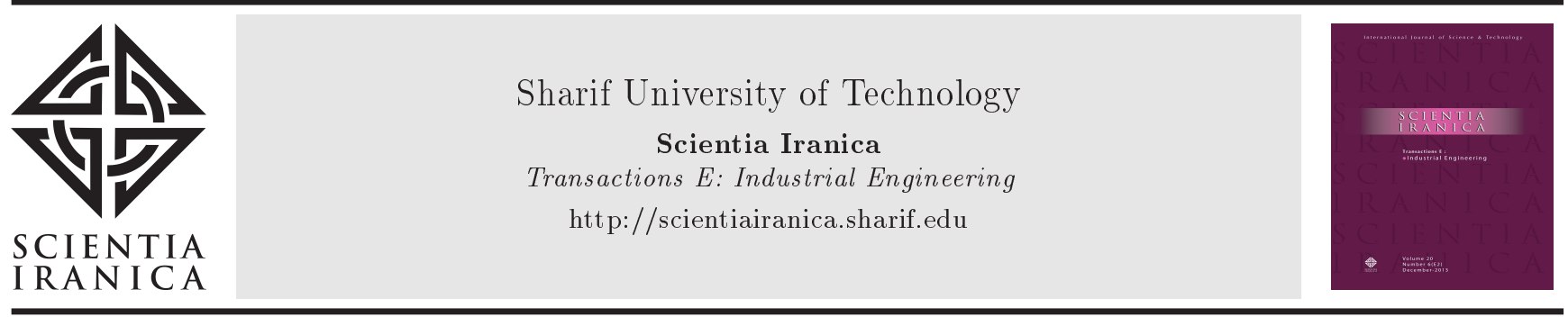

\title{
A septic B-spline collocation method for solving nonlinear singular boundary value problems arising in physiological models
}

\author{
A.R. Hadhoud ${ }^{a}$, K.K. Ali ${ }^{\mathrm{b}}$, and M.A. Shaalan ${ }^{\mathrm{c}, *}$ \\ a. Department of Mathematics, Faculty of Science, University of Menoufia, Shebein El-Koom, Egypt. \\ b. Department of Mathematics, Faculty of Science, University of Al-Azhar, Cairo, Nasr-City, Egypt. \\ c. Department of Basic Science, Higher Technological Institute, 10th of Ramadan City, Egypt.
}

Received 30 May 2018; received in revised form 1 October 2018; accepted 29 December 2018

\section{KEYWORDS \\ Septic B-spline collocation method; Singular boundary value problems; Absolute residual errors; \\ Error estimation; Uniform convergence.}

\begin{abstract}
In this paper, we present a numerical collocation method for nonlinear singular two-point boundary value problems of second order based on septic B-spline function. This method depends on different physiological processes such as steady-state oxygen diffusion in a spherical cell with the kinetics uptake of Michaelis-Menten and heat sources distribution in human head. The proposed method has uniform convergence for the exact solution. We will provide some physiological models proving that our method is very effective with acceptable maximum absolute errors and absolute residual errors.
\end{abstract}

(C) 2020 Sharif University of Technology. All rights reserved.

\section{Introduction}

Consider the nonlinear singular boundary value problems of the following form:

$$
z^{\prime \prime}(t)+\frac{k}{t} z^{\prime}(t)=g(t, z(t)), \quad t \in[0,1],
$$

under the boundary conditions:

$$
z^{\prime}(0)=0
$$

and:

$$
\alpha z(1)+\beta z^{\prime}(1)=\gamma,
$$

where $\alpha>0, \beta \geq 0, k \geq 1, \gamma \in R$ and $g(t, z(t))$ is a nonlinear continuous function with the partial derivative of $z$; also, $\frac{\partial g}{\partial z} \geq 0$, and it is continuous

\footnotetext{
*. Corresponding author.

E-mail addresses: adel_hadhoud2005@yahoo.com (A.R. Hadhoud); khalidkaram2012@yahoo.com (K.K. Ali); abozeid87@yahoo.com (M.A.Shaalan)
}

doi: $10.24200 /$ sci.2018.51012.1961 in the domain of $[0,1]$. In this paper, we introduce three models arising in physiology. The solutions to Eqs. (1)-(3) are unique ones in [1-3]. For $k=1$ and $g(t, z)=l e^{z(t)}$, where $l$ is a physical parameter, the first model arises in electro-hydrodynamics [4] following Eq. (1) under boundary conditions in Eqs. (2) and (3) with $\alpha=1, \beta=0$, and $\gamma=0$, in thermal explosions [5-8]. For different values of $k=0,1,2,3$ and $g(t, z)=\frac{m z(t)}{z(t)+c}$, where $m>0$, and $c>0$ in Eq. (1), the second model describes the steady-state oxygen diffusion in a spherical cell with the kinetics uptake of Michaelis-Menten [9-12]. The existence and uniqueness of the second model has been proved in [13]. Many authors studied the oxygen diffusion problem with:

$$
m=0.76129, \text { and } c=0.03119
$$

subject to the boundary conditions in Eqs. (2) and (3) with $\alpha=\gamma=5$, and $\beta=1$ [4-7]. The last model studies heat sources distribution in human head [14-16] at $k=2$ and $g(t, z)=-d e^{-p z(t)}$, where 
$d>0$, and $p>0$ in Eq. (1), under boundary conditions in Eqs. (2) and (3) with $\alpha=\gamma=0$, while $\beta$ takes three different values between $[4-6]$ and $[8,17]$. Many authors have introduced methods for solving a class of linear and nonlinear singular boundary value problems arising in applied science and engineering [1825]. Also, many applications of ordinary and partial differential equations arising in mechanical engineering, computer science, and electrical engineering have been investigated in [26-33].

Eq. (1) has a singular point at $t=0$. Thus, we first use L'Hôpital's rule to modify Eq. (1) at $t=0$ and then, convert the boundary value problems (1)-(3) to:

$$
\begin{aligned}
& z^{\prime \prime}(t)+r(t) z^{\prime}(t)=q(t, z(t)), \\
& z^{\prime}(0)=0, \quad \alpha z(1)+\beta z^{\prime}(1)=\gamma,
\end{aligned}
$$

where:

$$
\begin{aligned}
& r(t)= \begin{cases}0, & t=0, \\
\frac{k}{t}, & t \neq 0,\end{cases} \\
& q(t, z(t))= \begin{cases}\frac{g(t, z(t))}{k+1}, & t=0, \\
g(t, z(t)), & t \neq 0 .\end{cases}
\end{aligned}
$$

This paper is organized as follows. In Section 2, we analyze the proposed collocation method. In Section 3, the uniform convergence of the septic Bspline collocation method is derived. Section 4 enters into a discussion of some numerical models. Finally, in Section 5, conclusions are drawn.

\section{Septic B-spline collocation method}

Let $[0,1]$ be the domain of the proposed problem, which is divided into $n$ subintervals $\left\lfloor t_{j}, t_{j+1}\right\rfloor, j=$ $0,1, \ldots, n-1$ with equal step size of $h=\frac{1}{n}$ by the knots $t_{j}=j h,\{j=0,1, \ldots, n\}$, where $0=t_{0}<$ $t_{1}<\ldots<t_{n}=1$. We find additional knots $t_{-3}, t_{-2}, t_{-1}, t_{n+1}, t_{n+2}$, and $t_{n+3}$ outside the domain. Then, the septic B-spline function, $B_{j}(t)$, is introduced to find a numerical solution to the nonlinear singular boundary value problem (4); Eq. (5) is shown in Box I. Suppose $s(t)$ is the septic B-spline approximate solution to the exact solution $z(t)$ to problem (4), given by:

$$
s(t)=\sum_{j=-3}^{N+3} \delta_{j} B_{j}(t),
$$

where $\delta_{j}$ 's are constants found by the collocation points $t_{j}, j=0,1, \ldots, n$ and the boundary conditions. The septic B-spline function, $B_{j}(t)$, and its six derivatives $B_{j}^{\prime}(t), B_{j}^{\prime \prime}(t), B_{j}^{\prime \prime \prime}(t), B_{j}^{(4)}(t), B_{j}^{(5)}(t)$, and $B_{j}^{(6)}(t)$ at the knots are summarized in Table 1.

Based on Eqs. (5) and (6), the values of $s\left(t_{j}\right)$ at the nodal points and their six derivatives are:

$$
\begin{aligned}
s\left(t_{j}\right)= & \delta_{j-3}+120 \delta_{j-2}+1191 \delta_{j-1}+2416 \delta_{j} \\
& +1191 \delta_{j+1}+120 \delta_{j+2}+\delta_{j+3} \\
s^{\prime}\left(t_{j}\right)= & \frac{1}{h}\left(-7 \delta_{j-3}-392 \delta_{j-2}-1715 \delta_{j-1}\right. \\
& \left.+1715 \delta_{j+1}+392 \delta_{j+2}+7 \delta_{j+3}\right) \\
s^{\prime \prime}\left(t_{j}\right)= & \frac{1}{h^{2}}\left(42 \delta_{j-3}+1008 \delta_{j-2}+630 \delta_{j-1}\right. \\
& \left.-3360 \delta_{j}+630 \delta_{j+1}+1008 \delta_{j+2}+42 \delta_{j+3}\right) \\
s^{\prime \prime \prime}\left(t_{j}\right)= & \frac{1}{h^{3}}\left(-210 \delta_{j-3}-1680 \delta_{j-2}+3990 \delta_{j-1}\right.
\end{aligned}
$$
$B_{j}(t)=\frac{1}{h^{7}}\left\{\begin{array}{lr}\left(t-t_{j-4}\right)^{7} & t \in\left[t_{j-4}, t_{j-3}\right], \\ \left(t-t_{j-4}\right)^{7}-8\left(t-t_{j-3}\right)^{7} & t \in\left[t_{j-3}, t_{j-2}\right], \\ \left(t-t_{j-4}\right)^{7}-8\left(t-t_{j-3}\right)^{7}+28\left(t-t_{j-2}\right)^{7} & t \in\left[t_{j-2}, t_{j-1}\right], \\ \left(t-t_{j-4}\right)^{7}-8\left(t-t_{j-3}\right)^{7}+28\left(t-t_{j-2}\right)^{7}-56\left(t-t_{j-1}\right)^{7} \quad t \in\left[t_{j-1}, t_{j}\right], \\ \left(t_{j+4}-t\right)^{7}-8\left(t_{j+3}-t\right)^{7}+28\left(t_{j+2}-t\right)^{7}-56\left(t_{j+1}-t\right)^{7} t \in\left[t_{j}, t_{j+1}\right], \\ \left(t_{j+4}-t\right)^{7}-8\left(t_{j+3}-t\right)^{7}+28\left(t_{j+2}-t\right)^{7} & t \in\left[t_{j+1}, t_{j+2}\right], \\ \left(t_{j+4}-t\right)^{7}-8\left(t_{j+3}-t\right)^{7} & t \in\left[t_{j+2}, t_{j+3}\right], \\ \left(t_{j+4}-t\right)^{7} & t \in\left[t_{j+3}, t_{j+4}\right], \\ 0, & \text { otherwise. }\end{array}\right.$

$(j=-3,-2,-1,0, \cdots, N+2, N+3)$. 
Table 1. Nodal values of $B_{j}^{\prime}(t), B_{j}^{\prime \prime}(t), B_{j}^{\prime \prime \prime}(t), B_{j}^{(4)}(t), B_{j}^{(5)}(t)$, and $B_{j}^{(6)}(t)$.

\begin{tabular}{cccccccccc}
\hline & $\boldsymbol{t}_{\boldsymbol{j}-\boldsymbol{4}}$ & $\boldsymbol{t}_{\boldsymbol{j}-\mathbf{3}}$ & $\boldsymbol{t}_{\boldsymbol{j}-\mathbf{2}}$ & $\boldsymbol{t}_{\boldsymbol{j}-\mathbf{1}}$ & $\boldsymbol{t}_{\boldsymbol{j}}$ & $\boldsymbol{t}_{\boldsymbol{j}+\mathbf{1}}$ & $\boldsymbol{t}_{\boldsymbol{j}+\mathbf{2}}$ & $\boldsymbol{t}_{\boldsymbol{j}+\boldsymbol{3}}$ & $\boldsymbol{t}_{\boldsymbol{j}+\boldsymbol{4}}$ \\
\hline$B_{j}(t)$ & 0 & 1 & 120 & 1191 & 2416 & 1191 & 120 & 1 & 0 \\
$B_{j}^{\prime}(t)$ & 0 & $\frac{-7}{h}$ & $\frac{-392}{h}$ & $\frac{-1715}{h}$ & 0 & $\frac{1715}{h}$ & $\frac{392}{h}$ & $\frac{7}{h}$ & 0 \\
$B_{j}^{\prime \prime}(t)$ & 0 & $\frac{42}{h^{2}}$ & $\frac{1008}{h^{2}}$ & $\frac{630}{h^{2}}$ & $\frac{-3360}{h^{2}}$ & $\frac{630}{h^{2}}$ & $\frac{1008}{h^{2}}$ & $\frac{42}{h^{2}}$ & 0 \\
$B_{j}^{\prime \prime \prime}(t)$ & 0 & $\frac{-210}{h^{3}}$ & $\frac{-1680}{h^{3}}$ & $\frac{3990}{h^{3}}$ & 0 & $\frac{-3990}{h^{3}}$ & $\frac{1680}{h^{3}}$ & $\frac{210}{h^{3}}$ & 0 \\
$B_{j}^{(4)}(t)$ & 0 & $\frac{840}{h^{4}}$ & 0 & $\frac{-7560}{h^{4}}$ & $\frac{13440}{h^{4}}$ & $\frac{-7560}{h^{4}}$ & 0 & $\frac{840}{h^{4}}$ & 0 \\
$B_{j}^{(5)}(t)$ & 0 & $\frac{-2520}{h^{5}}$ & $\frac{10080}{h^{5}}$ & $\frac{-12600}{h^{5}}$ & 0 & $\frac{12600}{h^{5}}$ & $\frac{-10080}{h^{5}}$ & $\frac{2520}{h^{5}}$ & 0 \\
$B_{j}^{(6)}(t)$ & 0 & $\frac{5040}{h^{6}}$ & $\frac{-30240}{h^{6}}$ & $\frac{75600}{h^{6}}$ & $\frac{-100800}{h^{6}}$ & $\frac{75600}{h^{6}}$ & $\frac{-30240}{h^{6}}$ & $\frac{5040}{h^{6}}$ & 0 \\
\hline
\end{tabular}

$$
\begin{aligned}
& \left.-3990 \delta_{j+1}+1680 \delta_{j+2}+210 \delta_{j+3}\right) \\
s^{(4)}\left(t_{j}\right)= & \frac{1}{h^{4}}\left(840 \delta_{j-3}-7560 \delta_{j-1}+13440 \delta_{j}\right. \\
& \left.-7560 \delta_{j+1}+840 \delta_{j+3}\right) \\
s^{(5)}\left(t_{j}\right)= & \frac{1}{h^{5}}\left(-2520 \delta_{j-3}+10080 \delta_{j-2}-12600 \delta_{j-1}\right. \\
& \left.+12600 \delta_{j+1}-10080 \delta_{j+2}+2520 \delta_{j+3}\right) \\
s^{(6)}\left(t_{j}\right)= & \frac{1}{h^{6}}\left(5040 \delta_{j-3}-30240 \delta_{j-2}\right. \\
& +75600 \delta_{j-1}-100800 \delta_{j}+75600 \delta_{j+1} \\
& \left.-30240 \delta_{j+2}+5040 \delta_{j+3}\right)
\end{aligned}
$$

$$
\begin{aligned}
& \left.+1715 \delta_{j+1}+392 \delta_{j+2}+7 \delta_{j+3}\right)=g\left(t_{j}, z\left(t_{j}\right)\right), \\
& j=1,2, \ldots, n .
\end{aligned}
$$

After simplifying the above two equations, we get:

$$
\begin{aligned}
42 \delta_{-3} & +1008 \delta_{-2}+630 \delta_{-1}-3360 \delta_{0}+630 \delta_{1} \\
& +1008 \delta_{2}+42 \delta_{3}=h^{2} g_{0}, \\
\xi_{1}\left(t_{j}\right) & \delta_{j-3}+\xi_{2}\left(t_{j}\right) \delta_{j-2}+\xi_{3}\left(t_{j}\right) \delta_{j-1} \\
& +\xi_{4}\left(t_{j}\right) \delta_{j}+\xi_{5}\left(t_{j}\right) \delta_{j+1}+\xi_{6}\left(t_{j}\right) \delta_{j+2} \\
& +\xi_{7}\left(t_{j}\right) \delta_{j+3}=h^{2} t_{j} g_{j}, \\
j & =1,2, \ldots, n,
\end{aligned}
$$

where:

$$
\begin{aligned}
& g_{0}=\frac{g\left(t_{0}, z\left(t_{0}\right)\right)}{k+1}, \\
& \xi_{1}\left(t_{j}\right)=42 t_{j}-7 h, \quad \xi_{2}\left(t_{j}\right)=1008 t_{j}-392 h k, \\
& \xi_{3}\left(t_{j}\right)=630 t_{j}-1715 h, \quad \xi_{4}\left(t_{j}\right)=-3360 t_{j}, \\
& \xi_{5}\left(t_{j}\right)=630 t_{j}+1715 h, \quad \xi_{6}\left(t_{j}\right)=1008 t_{j}+392 h k, \\
& \xi_{7}\left(t_{j}\right)=42 t_{j}+7 h k, \quad g_{j}=g\left(t_{j}, z\left(t_{j}\right)\right), \\
& j=1,2, \ldots, n,
\end{aligned}
$$

and the boundary conditions are:

$$
\begin{aligned}
& -7 \delta_{-3}-392 \delta_{-2}-1715 \delta_{-1}+1715 \delta_{1}+392 \delta_{2} \\
& +7 \delta_{3}=0
\end{aligned}
$$

and:

$$
\begin{aligned}
& \lambda_{1}\left(t_{n}\right) \delta_{n-3}+\lambda_{2}\left(t_{n}\right) \delta_{n-2}+\lambda_{3}\left(t_{n}\right) \delta_{n-1} \\
& \quad+\lambda_{4}\left(t_{n}\right) \delta_{n}+\lambda_{5}\left(t_{n}\right) \delta_{n+1}+\lambda_{6}\left(t_{n}\right) \delta_{n+2} \\
& \quad+\lambda_{7}\left(t_{n}\right) \delta_{n+3}=h \gamma
\end{aligned}
$$

where: 


$$
\begin{aligned}
& \lambda_{1}\left(t_{n}\right)=h \alpha-7 \beta, \quad \lambda_{2}\left(t_{n}\right)=120 h \alpha-392 \beta, \\
& \lambda_{3}\left(t_{n}\right)=1191 h \alpha-1715 \beta, \quad \lambda_{4}\left(t_{n}\right)=2416 h \alpha, \\
& \lambda_{5}\left(t_{n}\right)=1191 h \alpha+1715 \beta, \\
& \lambda_{6}\left(t_{n}\right)=120 h \alpha+392 \beta, \quad \lambda_{7}\left(t_{n}\right)=h \alpha+7 \beta .
\end{aligned}
$$

However, still four equations are required. By differentiating Eq. (4) in $t$ fifth times, we get:

$$
\begin{aligned}
z^{(5)}(t) & +r(t) z^{(4)}(t)+3 r^{\prime}(t) z^{\prime \prime \prime}(t)+3 r^{\prime \prime}(t) z^{\prime \prime}(t) \\
& +r^{\prime \prime \prime}(t) z^{\prime}(t)=q_{1}(t, z(t)),
\end{aligned}
$$

where:

$$
\begin{aligned}
& r^{\prime}(t)=\left\{\begin{array}{ll}
0, & t=0, \\
\frac{-k}{t^{2}}, & t \neq 0,
\end{array} \quad r^{\prime \prime}(t)= \begin{cases}0, & t=0, \\
\frac{2 k}{t^{3}}, & t \neq 0,\end{cases} \right. \\
& r^{\prime \prime \prime}(t)= \begin{cases}0, & t=0, \\
\frac{-6 k}{t^{4}}, & t \neq 0,\end{cases}
\end{aligned}
$$

and:

$$
q_{1}(t, z(t))= \begin{cases}0, & t=0 \\ \frac{d g(t, z(t))}{d z} z^{\prime \prime \prime}(t) & \\ +3 \frac{d^{2} g(t, z(t))}{d z^{2}}, z^{\prime}(t) z^{\prime \prime}(t) & \\ +\frac{d^{3} g(t, z(t))}{d z^{3}}\left(z^{\prime}(t)\right)^{3}, & t \neq 0 .\end{cases}
$$

Putting $t=t_{0}$ in Eq. (12) and using Eq. (7), we have:

$$
\begin{gathered}
-2520 \delta_{-3}+10080 \delta_{-2}-12600 \delta_{-1}+12600 \delta_{1} \\
-10080 \delta_{2}+2520 \delta_{3}=0
\end{gathered}
$$

similarly, when $t=t_{n}$, we get:

$$
\begin{aligned}
\mu_{1}\left(t_{n}\right) & \delta_{n-3}+\mu_{2}\left(t_{n}\right) \delta_{n-2}+\mu_{3}\left(t_{n}\right) \delta_{n-1} \\
& +\mu_{4}\left(t_{n}\right) \delta_{n}+\mu_{5}\left(t_{n}\right) \delta_{n+1}+\mu_{6}\left(t_{n}\right) \delta_{n+2} \\
& +\mu_{7}\left(t_{n}\right) \delta_{n+3}=h^{5} \psi_{n},
\end{aligned}
$$

where:

$$
\begin{aligned}
\mu_{1}\left(t_{n}\right)= & -2520+840 h k+630 h^{2} k+252 h^{3} k \\
& +42 h^{4} k \\
\mu_{2}\left(t_{n}\right)= & 10080+5040 h^{2} k+6048 h^{3} k+2352 h^{4} k, \\
\mu_{3}\left(t_{n}\right)= & -12600-7560 h k-11970 h^{2} k \\
& +3780 h^{3} k+10290 h^{4} k, \\
\mu_{4}\left(t_{n}\right)= & 13440 h k-20160 h^{3} k, \\
\mu_{5}\left(t_{n}\right)= & 12600-7560 h k+11970 h^{2} k \\
& +3780 h^{3} k-10290 h^{4} k,
\end{aligned}
$$$$
\mu_{6}\left(t_{n}\right)=-10080-5040 h^{2} k+6048 h^{3} k
$$$$
-2352 h^{4} k
$$$$
\mu_{7}\left(t_{n}\right)=2520+840 h k-630 h^{2} k+252 h^{3} k
$$$$
-42 h^{4} k
$$

$$
\begin{gathered}
\psi_{n}=\frac{d g(t, z(t))}{d z} z^{\prime \prime \prime}(t)+3 \frac{d^{2} g(t, z(t))}{d z^{2}}, \\
z^{\prime}(t) z^{\prime \prime}(t)+\left.\frac{d^{3} g(t, z(t))}{d z^{3}}\left(z^{\prime}(t)\right)^{3}\right|_{t=t_{n}} .
\end{gathered}
$$

Differentiating Eq. (12) again with $t$, we get:

$$
\begin{aligned}
& z^{(6)}(t)+r(t) z^{(5)}(t)+2 r^{\prime}(t) z^{(4)}(t)+6 r^{\prime \prime}(t) \\
& z^{\prime \prime \prime}(t)+4 r^{\prime \prime \prime}(t) z^{\prime \prime}(t)+r^{(4)}(t) z^{\prime}(t) \\
& =q_{2}(t, z(t))
\end{aligned}
$$

where:

$$
r^{(4)}(t)= \begin{cases}0, & t=0 \\ \frac{24 k}{t^{5}}, & t \neq 0\end{cases}
$$

Also, we obtain $q_{2}(t, z(t))$ by the expression shown in Box II, where:

$$
q_{2}(t, z(t))=\left\{\begin{array}{cc}
\frac{d g(t, z(t))}{d z} z^{(4)}(t)+3 \frac{d^{2} g(t, z(t))}{d z^{2}}\left(z^{\prime \prime}(t)\right)^{2} & t=0 \\
1+\frac{k}{5} & \\
\frac{d g(t, z(t))}{d z} z^{(4)}(t)+\frac{d^{2} g(t, z(t))}{d z^{2}}\left(4 z^{\prime}(t)\right. & \left.z^{\prime \prime \prime}(t)+3\left(z^{\prime \prime}(t)\right)^{2}\right)+6 \frac{d^{3} g(t, z(t))}{d z^{3}}\left(z^{\prime}(t)\right)^{2} z^{\prime \prime}(t) \\
+\frac{d^{4} g(t, z(t))}{d z^{4}}\left(z^{\prime}(t)\right)^{4} & t \neq 0
\end{array}\right.
$$




$$
\begin{aligned}
& \left.z^{\prime \prime}(t)\right|_{t=0}=\frac{\left.g(t, z(t))\right|_{t=t_{0}}}{1+k} \text { and : } \\
& \left.z^{(4)}(t)\right|_{t=0}=\frac{\left.\left.\frac{d g(t, z(t))}{d z}\right|_{t=t_{0}} z^{\prime \prime}(t)\right|_{t=0}}{1+\frac{k}{3}} .
\end{aligned}
$$

Putting $t=t_{0}$ in Eq. (15) and using Eq. (7), we get:

$$
\begin{aligned}
5040 \delta_{-3}-30240 \delta_{-2}+75600 \delta_{-1}-100800 \delta_{0} \\
+75600 \delta_{1}+-30240 \delta_{2}+5040 \delta_{3}=h^{6} \sigma_{0},
\end{aligned}
$$

where:

$$
\sigma_{0}=\left.\frac{\frac{d g(t, z(t))}{d z} z^{(4)}(t)+3 \frac{d^{2} g(t, z(t))}{d z^{2}}\left(z^{\prime \prime}(t)\right)^{2}}{1+\frac{k}{5}}\right|_{t=t_{0}}
$$

Similarly, when $t=t_{n}$, we have:

$$
\begin{aligned}
\eta_{1}\left(t_{n}\right) & \delta_{n-3}+\eta_{2}\left(t_{n}\right) \delta_{n-2}+\eta_{3}\left(t_{n}\right) \delta_{n-1} \\
& +\eta_{4}\left(t_{n}\right) \delta_{n}+\eta_{5}\left(t_{n}\right) \delta_{n+1}+\eta_{6}\left(t_{n}\right) \delta_{n+2} \\
& +\eta_{7}\left(t_{n}\right) \delta_{n+3}=h^{6} \sigma_{n},
\end{aligned}
$$

where:

$$
\begin{aligned}
\eta_{1}\left(t_{n}\right)= & 5040-2520 h k-3360 h^{2} k \\
& -2520 h^{3} k-1008 h^{4} k-168 h^{5} k, \\
\eta_{2}\left(t_{n}\right)= & -30240+10080 h k-20160 h^{3} k \\
& -24192 h^{4} k-9408 h^{5} k,
\end{aligned}
$$

$$
\begin{aligned}
\eta_{3}\left(t_{n}\right)= & 75600-12600 h k+30240 h^{2} k \\
& +47880 h^{3} k-15120 h^{4} k-41160 h^{5} k, \\
\eta_{4}\left(t_{n}\right)= & -100800-53760 h^{2} k+80640 h^{4} k, \\
\eta_{5}\left(t_{n}\right)= & 75600+12600 h k+30240 h^{2} k \\
& -47880 h^{3} k-15120 h^{4} k+41160 h^{5} k, \\
\eta_{6}\left(t_{n}\right)= & -30240-10080 h k+20160 h^{3} k \\
& -24192 h^{4} k+9408 h^{5} k, \\
\eta_{7}\left(t_{n}\right)= & 5040+2520 h k-3360 h^{2} k \\
& +2520 h^{3} k-1008 h^{4} k+168 h^{5} k,
\end{aligned}
$$

and $\sigma_{n}$ is obtained by the expression shown in Box III. Then, from Eqs. (9)-(11), (13), (14), (16), and (17), we get a matrix in the following form:

$$
A T=B,
$$

where $A$ is non-singular square matrix $(n+7) \times(n+7)$ (as shown in Box IV), $T$ is dimensional vector $(n+7)$ with components $\delta_{j}$, and $B$ on the right-hand side is an $(n+7)$-dimensional vector as:

$$
\begin{aligned}
T= & \left(\begin{array}{lllllll}
\delta_{-3} & \delta_{-2} & \delta_{-1} & \delta_{0} & \delta_{1} & \ldots & \delta_{n-1} \\
& \delta_{n} & \delta_{n+1} & \delta_{n+2} & \delta_{n+3}
\end{array}\right)^{T}
\end{aligned}
$$

and:

$$
\sigma_{n}=\left.\left(\begin{array}{l}
\frac{d g(t, z(t))}{d z} z^{(4)}(t)+\frac{d^{2} g(t, z(t))}{d z^{2}}\left(4 z^{\prime}(t) z^{\prime \prime \prime}(t)+3\left(z^{\prime \prime}(t)\right)^{2}\right)+6 \frac{d^{3} g(t, z(t))}{d z^{3}}\left(z^{\prime}(t)\right)^{2} z^{\prime \prime}(t) \\
+\frac{d^{4} g(t, z(t))}{d z^{4}}\left(z^{\prime}(t)\right)^{4}
\end{array}\right)\right|_{t=t_{n}} .
$$

Box III

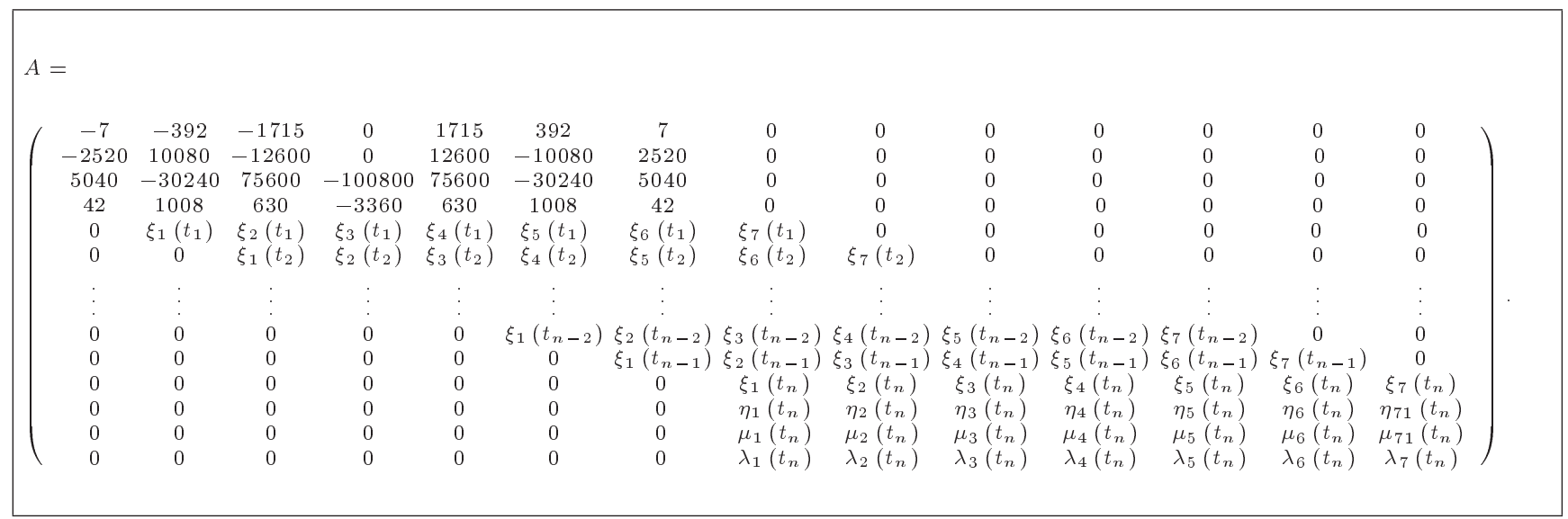




$$
B=\left(\begin{array}{lllllllllll}
0 & 0 & \sigma_{0} & g_{0} & g_{1} & \ldots & g_{n-1} & g_{n} & \sigma_{n} & \psi_{n} & \gamma
\end{array}\right)^{T}
$$

\section{Uniform convergence}

We estimate the truncation error for the septic B-spline collocation method in the interval $[a, b]$. Let $z(t)$ have continuous derivatives for all $t \in[a, b]$. Using Eq. (7), we get:

$$
\begin{aligned}
& s^{\prime}\left(t_{j-3}\right)+120 s^{\prime}\left(t_{j-2}\right) \\
& +1191 s^{\prime}\left(t_{j-1}\right)+2416 s^{\prime}\left(t_{j}\right)+1191 s^{\prime}\left(t_{j+1}\right) \\
& +120 s^{\prime}\left(t_{j+2}\right)+s^{\prime}\left(t_{j+3}\right)=\frac{1}{h}\left(-7 z\left(t_{j-3}\right)\right. \\
& -392 z\left(t_{j-2}\right)-1715 z\left(t_{j-1}\right)+1715 z\left(t_{j+1}\right) \\
& \left.+392 z\left(t_{j+2}\right)+7 s^{\prime}\left(t_{j+3}\right)\right) \\
& s^{\prime \prime}\left(t_{j-3}\right)+120 s^{\prime \prime}\left(t_{j-2}\right)+1191 s^{\prime \prime}\left(t_{j-1}\right) \\
& +2416 s^{\prime \prime}\left(t_{j}\right)+1191 s^{\prime \prime}\left(t_{j+1}\right)+120 s^{\prime \prime}\left(t_{j+2}\right) \\
& +s^{\prime \prime}\left(t_{j+3}\right)=\frac{1}{h^{2}}\left(42 z\left(t_{j-3}\right)+1008 z\left(t_{j-2}\right)\right. \\
& +630 z\left(t_{j-1}\right)-3360 z\left(t_{j}\right)+630 z\left(t_{j+1}\right) \\
& \left.+1008 z\left(t_{j+2}\right)+42 z\left(t_{j+3}\right)\right) \\
& s^{\prime \prime \prime}\left(t_{j-3}\right)+120 s^{\prime \prime \prime}\left(t_{j-2}\right)+1191 s^{\prime \prime \prime}\left(t_{j-1}\right) \\
& +2416 s^{\prime \prime \prime}\left(t_{j}\right)+1191 s^{\prime \prime \prime}\left(t_{j+1}\right)+120 s^{\prime \prime \prime}\left(t_{j+2}\right) \\
& +s^{\prime \prime \prime}\left(t_{j+3}\right)=\frac{1}{h^{3}}\left(-210 z\left(t_{j-3}\right)-1680 z\left(t_{j-2}\right)\right. \\
& +3990 z\left(t_{j-1}\right)-3990 z\left(t_{j+1}\right)+1680 z\left(t_{j+2}\right) \\
& \left.+210 z\left(t_{j+3}\right)\right) \\
& s^{(4)}\left(t_{j-3}\right)+120 s^{(4)}\left(t_{j-2}\right)+1191 s^{(4)}\left(t_{j-1}\right) \\
& +2416 s^{(4)}\left(t_{j}\right)+1191 s^{(4)}\left(t_{j+1}\right) \\
& +120 s^{(4)}\left(t_{j+2}\right)+s^{(4)}\left(t_{j+3}\right)=\frac{1}{h^{4}}\left(840 z\left(t_{j-3}\right)\right. \\
& -7560 z\left(t_{j-1}\right)+13440 z\left(t_{j}\right)-7560 z\left(t_{j+1}\right) \\
& \left.+840 z\left(t_{j+3}\right)\right)
\end{aligned}
$$

$$
\begin{aligned}
& s^{(5)}\left(t_{j-3}\right)+120 s^{(5)}\left(t_{j-2}\right)+1191 s^{(5)}\left(t_{j-1}\right) \\
& \quad+2416 s^{(5)}\left(t_{j}\right)+1191 s^{(5)}\left(t_{j+1}\right) \\
& \quad+120 s^{(5)}\left(t_{j+2}\right)+s^{(5)}\left(t_{j+3}\right) \\
& \quad=\frac{1}{h^{5}}\left(-2520 z\left(t_{j-3}\right)+10080 z\left(t_{j-2}\right)\right. \\
& \quad-12600 z\left(t_{j-1}\right)+12600 z\left(t_{j+1}\right)-10080 z\left(t_{j+2}\right) \\
& \left.\quad+2520 z\left(t_{j+3}\right)\right), \\
& s^{(6)}\left(t_{j-3}\right)+120 s^{(6)}\left(t_{j-2}\right)+1191 s^{(6)}\left(t_{j-1}\right) \\
& \quad+2416 s^{(6)}\left(t_{j}\right)+1191 s^{(6)}\left(t_{j+1}\right) \\
& \quad+120 s^{(6)}\left(t_{j+2}\right)+s^{(6)}\left(t_{j+3}\right)=\frac{1}{h^{6}}\left(5040 z\left(t_{j-3}\right)\right. \\
& \quad+30240 z\left(t_{j-2}\right)+75600 z\left(t_{j-1}\right)-100800 z\left(t_{j}\right) \\
& +75600 z\left(t_{j+1}\right)-30240 z\left(t_{j+2}\right) \\
& \left.+5040 z\left(t_{j+3}\right)\right) . \\
& \\
& +
\end{aligned}
$$

Using the operator notation $E\left(z\left(t_{j}\right)\right)=z\left(t_{j+1}\right)$ [34], Eqs. (19)-(24) are expressed by Eqs. (25)-(30) as shown in Box V. In Eqs. (25)-(30), putting the operator notation $E=e^{h D}$ as the expansion to the power of $h D$, we have:

$$
\begin{aligned}
s^{\prime}\left(t_{j}\right)= & z^{\prime}\left(t_{j}\right)-\frac{h^{8}}{151200} z^{(9)}\left(t_{j}\right)+\frac{h^{10}}{399168} z^{(11)}\left(t_{j}\right) \\
& +O\left(h^{11}\right), \\
s^{\prime \prime}\left(t_{j}\right)= & z^{\prime \prime}\left(t_{j}\right)-\frac{h^{6}}{30240} z^{(8)}\left(t_{j}\right)+\frac{h^{8}}{60480} z^{(10)}\left(t_{j}\right) \\
& -\frac{h^{10}}{532224} z^{(12)}\left(t_{j}\right)+O\left(h^{11}\right), \\
s^{\prime \prime \prime}\left(t_{j}\right)= & z^{\prime \prime \prime}\left(t_{j}\right)+\frac{h^{6}}{6048} z^{(9)}\left(t_{j}\right)-\frac{h^{8}}{33600} z^{(11)}\left(t_{j}\right) \\
& +\frac{h^{10}}{295680} z^{(13)}\left(t_{j}\right)+O\left(h^{11}\right), \\
s^{(4)}\left(t_{j}\right)= & z^{(4)}\left(t_{j}\right)+\frac{h^{4}}{720} z^{(8)}\left(t_{j}\right)-\frac{h^{6}}{3024} z^{(10)}\left(t_{j}\right) \\
& +\frac{17 h^{8}}{604800} z^{(12)}\left(t_{j}\right)-\frac{h^{10}}{997920} z^{(14)}\left(t_{j}\right) \\
& +O\left(h^{11}\right),
\end{aligned}
$$




$$
\begin{aligned}
& s^{\prime}\left(t_{j}\right)=\frac{1}{h}\left(\frac{-7 E^{-3}-392 E^{-2}-1715 E^{-1}+1715 E+392 E^{2}+7 E^{3}}{E^{-3}+120 E^{-2}+1191 E^{-1}+2461+1191 E+120 E^{2}+E^{3}}\right) z\left(t_{j}\right), \\
& s^{\prime \prime}\left(t_{j}\right)=\frac{1}{h^{2}}\left(\frac{42 E^{-3}+1008 E^{-2}+630 E^{-1}-3360+630 E+1008 E^{2}+42 E^{3}}{E^{-3}+120 E^{-2}+1191 E^{-1}+2461+1191 E+120 E^{2}+E^{3}}\right) z\left(t_{j}\right), \\
& s^{\prime \prime \prime}\left(t_{j}\right)=\frac{1}{h^{3}}\left(\frac{-210 E^{-3}-1680 E^{-2}+3990 E^{-1}-3990 E+1680 E^{2}+210 E^{3}}{E^{-3}+120 E^{-2}+1191 E^{-1}+2461+1191 E+120 E^{2}+E^{3}}\right) z\left(t_{j}\right), \\
& s^{(4)}\left(t_{j}\right)=\frac{1}{h^{4}}\left(\frac{840 E^{-3}-7560 E^{-1}+13440-7560 E+840 E^{3}}{E^{-3}+120 E^{-2}+1191 E^{-1}+2461+1191 E+120 E^{2}+E^{3}}\right) z\left(t_{j}\right), \\
& s^{(5)}\left(t_{j}\right)=\frac{1}{h^{5}}\left(\frac{-2520 E^{-3}+10080 E^{-2}-12600 E^{-1}+12600 E-10080 E^{2}+2520 E^{3}}{E^{-3}+120 E^{-2}+1191 E^{-1}+2461+1191 E+120 E^{2}+E^{3}}\right) z\left(t_{j}\right), \\
& s^{(6)}\left(t_{j}\right)=\frac{1}{h^{6}}\left(\frac{5040 E^{-3}-30240 E^{-2}+75600 E^{-1}-100800+75600 E-30240 E^{2}+5040 E^{3}}{E^{-3}+120 E^{-2}+1191 E^{-1}+2461+1191 E+120 E^{2}+E^{3}}\right) z\left(t_{j}\right) .
\end{aligned}
$$

\section{Box V}

$$
\begin{aligned}
s^{(5)}\left(t_{j}\right)= & z^{(5)}\left(t_{j}\right)-\frac{h^{4}}{240} z^{(9)}\left(t_{j}\right)+\frac{h^{6}}{3024} z^{(11)}\left(t_{j}\right) \\
& -\frac{11 h^{8}}{604800} z^{(13)}\left(t_{j}\right)+\frac{13 h^{10}}{665280} z^{(15)}\left(t_{j}\right) \\
& +O\left(h^{11}\right), \\
s^{(6)}\left(t_{j}\right)= & z^{(6)}\left(t_{j}\right)-\frac{h^{2}}{12} z^{(8)}\left(t_{j}\right)+\frac{h^{4}}{240} z^{(10)}\left(t_{j}\right) \\
& -\frac{h^{6}}{6048} z^{(12)}\left(t_{j}\right)+\frac{h^{8}}{210600} z^{(14)}\left(t_{j}\right) \\
+ & \frac{101 h^{10}}{159667200} z^{(16)}\left(t_{j}\right)+O\left(h^{11}\right) .
\end{aligned}
$$

Theorem 1. Let the nonlinear singular two-point boundary value problems of the second order have the form of Eqs. (1)-(3) with the exact solution, $z(t)$, and approximate solution, $s(t)$. Then, the septic B-spline collocation method has a truncation error of $O\left(h^{8}\right)$ and the convergence of this method is $O\left(h^{6}\right)$ for sufficiently small.

\section{Numerical problems and discussion}

We introduce some physiological applications related to nonlinear singular two-point boundary value problems of the second order. The results are generated with Mathematica using FindRoot function. The absolute value of the difference between the exact solution and the numerical solution is calculated and the absolute residual errors for the problems with no exact solutions are found.

Problem 1. Consider the following nonlinear singular boundary value problem [5]:

$$
\begin{aligned}
& z^{\prime \prime}(t)+\frac{1}{t} z^{\prime}(t)=-e^{z(t)}, \\
& z^{\prime}(0)=0, \quad z(1)=0
\end{aligned}
$$

where the exact solution to Model (38) takes the form $z(t)=2 \log \left(\frac{A+1}{A t^{2}+1}\right)$, with $A=3-2 \sqrt{2}$. Table 2 shows 
Table 2. Values of the maximum absolute errors for Problem 1.

\begin{tabular}{cccccccccc}
\hline $\boldsymbol{n}$ & Method in [6] & $\boldsymbol{n}$ & Method in [5] & $\boldsymbol{n}$ & Method in [7] & $\boldsymbol{n}$ & Method in [8] & $\boldsymbol{n}$ & Present method \\
\hline 20 & $3.16 \times 10^{-5}$ & 6 & $4.00 \times 10^{-4}$ & 20 & $5.03 \times 10^{-10}$ & 16 & $3.11 \times 10^{-6}$ & 10 & $1.08 \times 10^{-10}$ \\
90 & $1.55 \times 10^{-6}$ & 8 & $2.53 \times 10^{-5}$ & 40 & $4.11 \times 10^{-12}$ & 32 & $2.35 \times 10^{-7}$ & 20 & $7.10 \times 10^{-13}$ \\
161 & $4.90 \times 10^{-7}$ & 10 & $2.10 \times 10^{-6}$ & 90 & $2.54 \times 10^{-14}$ & 64 & $1.50 \times 10^{-8}$ & 40 & $6.05 \times 10^{-15}$ \\
\hline
\end{tabular}

Table 3. Numerical results for Problem 2.

\begin{tabular}{ccccc}
\hline $\boldsymbol{t}$ & $\begin{array}{c}\text { Method in [6] } \\
\boldsymbol{n}=\mathbf{6 0}\end{array}$ & $\begin{array}{c}\text { Method in [7] } \\
\boldsymbol{n}=\mathbf{6 0}, \boldsymbol{j}_{\boldsymbol{u}}=\mathbf{5}\end{array}$ & $\begin{array}{c}\text { Present method } \\
\boldsymbol{n}=\mathbf{2 0}\end{array}$ & $\begin{array}{c}\text { Present method } \\
\boldsymbol{n}=\mathbf{6 0}\end{array}$ \\
\hline 0.0 & 0.82848327295802 & - & 0.828483290367166 & 0.8284832903611578 \\
0.1 & 0.82970607521884 & 0.829706075229 & 0.829706092441288 & 0.8297060924352763 \\
0.2 & 0.83337471691089 & 0.833374716949 & 0.8333757335984896 & 0.8333747335924774 \\
0.3 & 0.83948989814383 & 0.839489898181 & 0.8394899139611924 & 0.8394899139551798 \\
0.4 & 0.84805277036165 & 0.848052770408 & 0.8480527850035551 & 0.8480527849975414 \\
0.5 & 0.85906491397434 & 0.859064914012 & 0.8590649271767254 & 0.8590649271707098 \\
0.6 & 0.87252830841853 & 0.872528308451 & 0.8725283199657823 & 0.872528319959764 \\
0.7 & 0.88844529589927 & 0.888445295928 & 0.8884453056306942 & 0.8884453056246733 \\
0.8 & 0.90681854026297 & 0.906818540286 & 0.9068185480743092 & 0.9068185480682849 \\
0.9 & 0.92765098252660 & 0.927650982542 & 0.9276509883730854 & 0.9276509883970594 \\
1.0 & 0.95094579461056 & 0.950945794637 & 0.9509457985037988 & 0.9509457984979333 \\
\hline
\end{tabular}

that the maximum absolute errors of the septic B-spline collocation method are acceptable in comparison with other methods [5-8].

Problem 2. Consider another nonlinear singular boundary value problem [5] as follows:

$$
\begin{aligned}
& z^{\prime \prime}(t)+\frac{2}{t} z^{\prime}(t)=\frac{0.76129 z(t)}{z(t)+0.03119}, \\
& z^{\prime}(0)=0, \quad 5 z(1)+z^{\prime}(1)=5,
\end{aligned}
$$

Table 3 and Figure 1 show the numerical solutions of the present method in comparison with those of other methods [6,7]. Also, Figure 2 illustrates the absolute residual errors $R_{n}(t)=\mid z^{\prime \prime}(t)+\frac{2}{t} z^{\prime}(t)-$ $\frac{0.76129 z(t)}{z(t)+0.03119} \mid, 0<t \leq 1$, at $n=60$ for Model (39), which does not have any exact solution.

Problem 3. Consider the following nonlinear singular boundary value problem [5]:

$$
z^{\prime \prime}(t)+\frac{2}{t} z^{\prime}(t)=-e^{-z(t)}
$$

subject to boundary conditions in three cases:
(i) $z^{\prime}(0)=0, \quad 0.1 z(1)+z^{\prime}(1)=0$,
(ii) $z^{\prime}(0)=0, \quad z(1)+z^{\prime}(1)=0$,
(iii) $z^{\prime}(0)=0, \quad 2 z(1)+z^{\prime}(1)=0$.

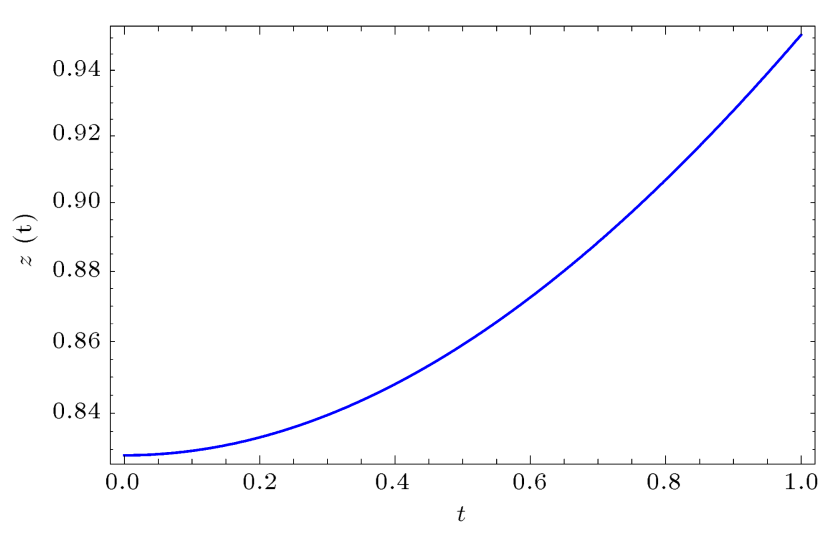

Figure 1. Numerical solutions to Problem 2 by the present method for $n=40$.

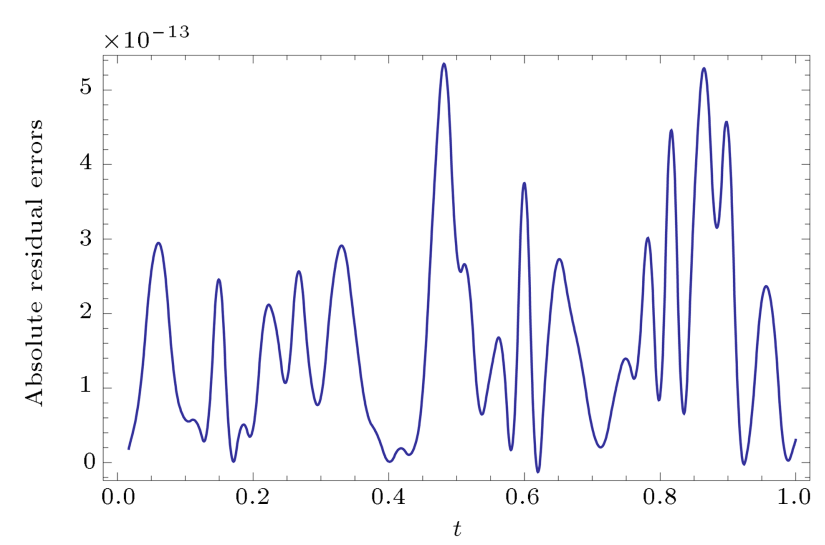

Figure 2. Absolute residual errors for Problem 2 at $n=60$. 
Table 4. Numerical results for Problem 3.

\begin{tabular}{ccccc}
\hline & Method in [6] & Present method & Method in [17] & Present method \\
$\boldsymbol{t}$ & $\boldsymbol{\alpha}=\mathbf{0 . 1}, \boldsymbol{\beta}=\mathbf{1}$, & $\boldsymbol{\alpha}=\mathbf{0 . 1}, \boldsymbol{\beta}=\mathbf{1}$, & $\boldsymbol{\alpha}=\mathbf{1}, \boldsymbol{\beta}=\mathbf{1}$, & $\boldsymbol{\alpha}=\mathbf{1 , \boldsymbol { \beta } = \mathbf { 1 } ,}$ \\
& $\boldsymbol{\gamma}=\mathbf{0 , \boldsymbol { n } = \mathbf { 6 0 }}$ & $\boldsymbol{\gamma}=\mathbf{0 , \boldsymbol { n } = \mathbf { 4 0 }}$ & $\boldsymbol{\gamma}=\mathbf{0 , \boldsymbol { n } = \mathbf { 4 0 }}$ & $\boldsymbol{\gamma}=\mathbf{0 , \boldsymbol { n } = \mathbf { 4 0 }}$ \\
\hline 0.0 & 1.14703993670271 & 1.147039019329926 & 0.3675181074 & 0.3675168151351779 \\
0.1 & 1.14651055946170 & 1.1465096424107886 & 0.3663637561 & 0.36636232924657036 \\
0.2 & 1.14492141825538 & 1.1449205020922848 & 0.3628959378 & 0.362894066125307 \\
0.3 & 1.14226947822689 & 1.1422685635712044 & 0.3570991429 & 0.3570975457273243 \\
0.4 & 1.13854966085306 & 1.1385487483652494 & 0.3489499903 & 0.3489484206280108 \\
0.5 & 1.13375481292594 & 1.1337539033259227 & 0.3384136581 & 0.3384121487566203 \\
0.6 & 1.12787566262296 & 1.1278747567071357 & 0.3254450019 & 0.3254435224405896 \\
0.7 & 1.12090076206338 & 1.120899860725792 & 0.3099878567 & 0.309986040238538 \\
0.8 & 1.11281641561478 & 1.112815519868545 & 0.2919789654 & 0.29197110306288504 \\
0.9 & 1.10360659299888 & 1.103605704000184 & 0.2713185637 & 0.2713170101649496 \\
1.0 & 1.09325282603337 & 1.0932519451088027 & 0.2479292837 & 0.24792772332382068 \\
\hline
\end{tabular}

Table 5. Absolute residual errors and numerical results for Problem 3.

\begin{tabular}{|c|c|c|c|c|}
\hline$t$ & $\begin{array}{l}\text { Method in [5] } \\
\alpha=2, \beta=1, \\
\gamma=0, n=10\end{array}$ & $\begin{array}{l}\text { Method in }[8] \\
\alpha=2, \beta=1, \\
\gamma=0, n=10\end{array}$ & $\begin{array}{c}\text { Present method } \\
\alpha=2, \beta=1 \\
\gamma=0, n=10\end{array}$ & $\begin{array}{c}\text { Present method } \\
\alpha=2, \beta=1 \\
\gamma=0, n=40\end{array}$ \\
\hline 0.0 & - & - & - & 0.27002964789651424 \\
\hline 0.1 & $8.2261 \mathrm{E}-04$ & $1.17552 \mathrm{E}-05$ & $1.4627 \times 10^{-12}$ & 0.26875690062949353 \\
\hline 0.2 & $7.7789 \mathrm{E}-04$ & $5.59474 \mathrm{E}-06$ & $1.37901 \times 10^{-12}$ & 0.2649328175381816 \\
\hline 0.3 & $7.2546 \mathrm{E}-04$ & $1.77891 \mathrm{E}-06$ & $1.26776 \times 10^{-12}$ & 0.258539789381345 \\
\hline 0.4 & $6.5808 \mathrm{E}-04$ & $7.15070 \mathrm{E}-07$ & $1.11477 \times 10^{-12}$ & 0.2495481802537009 \\
\hline 0.5 & $5.8074 \mathrm{E}-04$ & $4.01100 \mathrm{E}-07$ & $9.49685 \times 10^{-13}$ & 0.23791588758927304 \\
\hline 0.6 & $4.9869 \mathrm{E}-04$ & $1.37600 \mathrm{E}-06$ & $7.78599 \times 10^{-13}$ & 0.22358770718136314 \\
\hline 0.7 & $4.1676 \mathrm{E}-04$ & $2.70372 \mathrm{E}-06$ & $6.17506 \times 10^{-13}$ & 0.20649448302373294 \\
\hline 0.8 & $3.3896 \mathrm{E}-04$ & $6.88321 \mathrm{E}-06$ & $4.69846 \times 10^{-13}$ & 0.18655201416659845 \\
\hline 0.9 & $2.6816 \mathrm{E}-04$ & $1.83404 \mathrm{E}-05$ & $3.44058 \times 10^{-13}$ & 0.16365968158038072 \\
\hline 1.0 & $2.0607 \mathrm{E}-04$ & $2.53189 \mathrm{E}-05$ & $2.41918 \times 10^{-13}$ & 0.137698746613583 \\
\hline
\end{tabular}

Table 4 and Figure 3 show the numerical solutions of the present method with different values of $\alpha$ in comparison with other methods [6,17]. Furthermore, Table 5 shows the absolute residual errors $R_{n}(t)=$ $\left|z^{\prime \prime}(t)+\frac{2}{t} z^{\prime}(t)+e^{-z(t)}\right|, 0<t \leq 1$, at $\alpha=2, n=10$ and indicates that numerical solutions of Model (40) at $\alpha=2, n=40$ do not have any exact solution.

\section{Conclusion}

We presented a septic B-spline collocation method for finding numerical solutions to the nonlinear singular two-point boundary value problems of the second order. At different values of $n$, the numerical results showed that the proposed method had efficient solutions for the studied models. Three applications were investigated, which played a vital role in physiological models. We

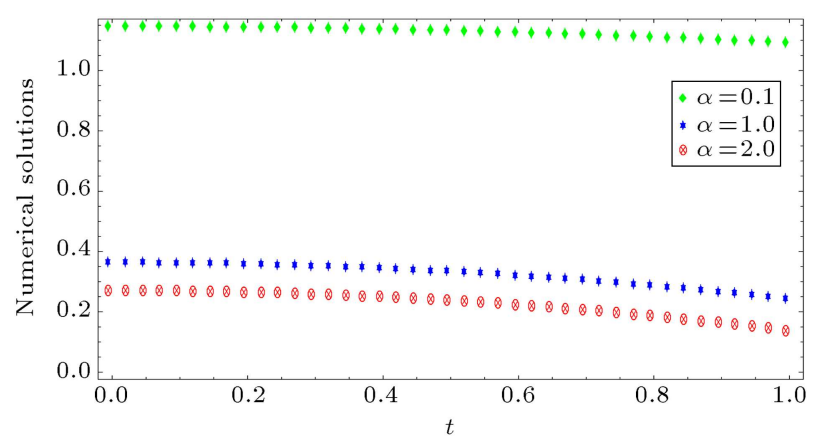

Figure 3. Numerical solutions to Problem 3 at different values of $\alpha$.

observed that numerical accuracy of the proposed method for the thermal explosions problem was better than the results obtained by [5-8] and for the problems of oxygen diffusion in a spherical cell with the kinetics 
uptake of Michaelis-Menten and heat sources distribution in human head, numerical solutions were similar to the results obtained by [5-8] and [17]. Nevertheless, it should be noted that these problems do not have exact solutions. Accordingly, we calculated the absolute residual errors and presented the results.

\section{Acknowledgments}

The authors are grateful to the reviewers for their careful reading of the manuscript as well as precious suggestions and comments.

\section{References}

1. Chawla, M.M. and Shivkumar, P.N. "On the existence of solutions of a class of singular two-point nonlinear boundary value problems", J. Comput. Appl. Math., 19, pp. 379-388 (1987).

2. Russell, R.D. and Shampine, L.F. "Numerical methods for singular boundary value problems", SIAM J. Numer. Anal., 12, pp. 13-36 (1975).

3. Ford, W.F. and Pennline, J.A. "Singular non-linear two-point boundary value problems: Existence and uniqueness", Nonlinear Anal., 71, pp. 1059-1072 (2009).

4. Khuri, S. and Sayfy, A. "A novel approach for the solution of a class of singular boundary value problems arising in physiology", Math. Comput. Modelling, 52, pp. 626-636 (2010).

5. Singh, R. and Kumar, J. "An efficient numerical technique for the solution of nonlinear singular boundary value problems", Comput. Phys. Comm., 185, pp. 1282-1289 (2014).

6. Caglar, H., Caglar, N., and Ozer, M. "B-spline solution of non-linear singular boundary value problems arising in physiology", Chaos Solitons Fractals, 39(3), pp. 1232-1237 (2009).

7. Sahlan, M.N. and Hashemizadeh, E. "Wavelet Galerkin method for solving nonlinear singular boundary value problems arising in physiology", Applied Mathematics and Computation, 250, pp. 260-269 (2015).

8. Niu, J., Xu, M., Lin, Y., et al. "Numerical solution of nonlinear singular boundary value problems", Journal of Computational and Applied Mathematics, 331, pp. 42-51 (2018).

9. Lin, S.H. "Oxygen diffusion in a spherical cell with nonlinear oxygen uptake kinetics", J. Theor. Biol., 60, pp. 449-457 (1976).

10. McElwain, D.L.S. "A re-examination of oxygen diffusion in a spherical cell with Michaelis-Menten oxygen uptake kinetics", J. Theor. Biol., 71, pp. 255-263 (1978).
11. Wazwaz, A. "The variational iteration method for solving nonlinear singular boundary value problems arising in various physical models", Commun. Nonlinear Sci. Numer. Simul., 16, pp. 3881-3886 (2011).

12. Simpson, M.J. and Ellery, A.J. "An analytical solutions for diffusion and nonlinear uptake of oxygen in a spherical cell", Applied Mathematical Modelling, 36, pp. 3329-3334 (2012).

13. Hiltmann, P. and Lory, P. "On oxygen diffusion in a spherical cell with Michaelis-Menten oxygen uptake kinetics", Bull. Math. Biol., 45, pp. 661-664 (1983).

14. Flesch, U. "The distribution of heat sources in the human head: a theoretical consideration", J. Theor. Biol., 54, pp. 285-287 (1975).

15. Duggan, R. and Goodman, A. "Pointwise bounds for a nonlinear heat conduction model of the human head", Bull. Math. Biol., 48(2), pp. 229-236 (1986).

16. Garner, J.B. and Shivaji, R. "Diffusion problems with mixed nonlinear boundary condition", J. Math. Anal. Appl., 148, pp. 422-430 (1990).

17. Rashidinia, J., Mohammadi, R., and Jalilian, R. "The numerical solution of nonlinear singular boundary value problems arising in physiology", Applied Mathematics and Computation, 185, pp. 360-367 (2007).

18. Khuri, S.A. and Sayfy, A. "A mixed decompositionspline approach for the numerical solution of a class of singular boundary value problems", Applied Mathematical Modelling, 40, pp. 4664-468 (2016).

19. Roul, P. and Thula, K. "A new high-order numerical method for solving singular two-point boundary value problems", Journal of Computational and Applied Mathematics, 343, pp. 556-574 (2018). https://doi.org/10.1016/j.cam.2018.04.056

20. Khuri, S.A. and Sayfy, A. "Numerical solution for the nonlinear Emden-Fowler type equations by a fourthorder adaptive method", Int. J. Comput. Methods, 11(1) (2014).

21. Roul, P. and Ujwal, W. "A novel numerical approach and its convergence for numerical solution of nonlinear doubly singular boundary value problems", J. Compt. Appl. Math., 296, pp. 661-676 (2016).

22. Roul, P. and Kiran, T. "A fourth order B-spline collocation method and its error analysis for Bratutype and Lane-Emden problems", Int. J. Comp. Math., 96(1), pp. 85-104 (2017).

23. Pirabaharan, P. and Chandrakumar, R.D. "A computational method for solving a class of singular boundary value problems arising in science and engineering", Egyptian Journal of Basic and Applied Sciences, 3, pp. 383-391 (2016).

24. Nasab, A.K., Kılıcman, A., Babolian, E., et al. "Wavelet analysis method for solving linear and nonlinear singular boundary value problems", Applied Mathematical Modelling, 37, pp. 5876-5886 (2013).

25. Chang, S.H. "Taylor series method for solving a class of nonlinear singular boundary value problems arising 
in applied science", Applied Mathematics and Computation, 235, pp. 110-117 (2014).

26. Goh, J. and Ali, N.H.M. "New high-accuracy nonpolynomial spline group explicit iterative method for two-dimensional elliptic boundary value problems", Scientia Iranica D, 24(6), pp. 3181-3192 (2017).

27. Rashidinia, J., Mohammadi, R., and Jalilian, R. "Quintic spline solution of boundary value problems in the plate deflection theory", Scientia Iranica D, 16(1), pp. 53-59 (2009).

28. Rashidinia, J., Jalilian, R., and Mohammadi, R. "Convergence analysis of spline solution of certain twopoint boundary value problems", Scientia Iranica D, 16(2), pp. 128-136 (2009).

29. Ak, T., Karakoc, S.B.G., and Biswas, A. "Application of Petrov-Galerkin finite element method to shallow water waves model: Modified Korteweg-deVries equation", Scientia Iranica B, 24(3), pp. 1148-1159 (2017).

30. Triki, H., Ak, T., Moshokoa, S., et al. "Soliton solutions to KdV equation with spatio-temporal dispersion", Ocean Engineering, 114, pp. 192-203 (2016).

31. AK, T., Aydemir, T., Saha, A., et al. "Propagation of nonlinear shock waves for the generalised Oskolkov equation and its dynamic motions in the presence of an external periodic perturbation", Pramana-J. Phys., 90(6) (2018).

32. Ak, T. and Karakoc, S.B.G. "A numerical technique based on collocation method for solving modified Kawahara equation", Journal of Ocean Engineering and Science, 3(1), pp. 67-75 (2018).

33. Ak, T., Dhawan, S., Karakoc, S.B.G., et al. "Numerical study of Rosenau-KdV equation using finite element method based on collocation approach", Mathematical Modelling and Analysis, 22(3), pp. 373-388 (2017).
34. Sastry, S.S., Introductory Methods of Numerical Analysis, PHI Learning Pvt, Ltd (2012).

\section{Biographies}

Adel Rashad Hadhoud graduated from Menoufia University in 2002 with a BSc degree in Mathematics. He received his $\mathrm{MSc}$ and $\mathrm{PhD}$ degrees in Pure Mathematics from the same university in 2008 and 2012 , respectively. He is currently a lecturer in the Department of Mathematics at Menoufia University, Shebein El-Koom, Egypt. He has carried out research on partial differential equations, numerical analysis, and applied mathematics and has some publications in these areas.

Khalid Karam Ali graduated from Al-Azhar University in 2005 with a BSc degree in Mathematics. He received his $\mathrm{MSc}$ and $\mathrm{PhD}$ degrees in Pure Mathematics from the same university in 2015 and 2018, respectively. He is currently a lecturer in the Department of Mathematics at Al-Azhar University, Cairo, Nasr City, Egpyt. He has published more than 25 papers in international journals. His areas of research interest are finite element method, numerical simulation, solitary waves, and applied mathematics.

Mohamed Abozeid Shaalan is working as an assistant lecturer in the Department of Basic Science at Higher Technological Institute, Ramadan City, Egypt. He earned his BSc and MSc degrees in Mathematics from Zagazig University, Egypt, in 2007 and 2014, respectively. His research interests include numerical analysis, matrix differential equations, B-Spline functions, and fluid dynamics. 